\title{
An Overview of First 100 Cardiac Surgery Cases at a Newly Developed Satellite Center in Sukkur, Pakistan
}

Kashif Zia ${ }^{1}$, Ali R. Mangi ${ }^{1}$, Syed Minhaj ${ }^{1}$, Khuzaima Tariq ${ }^{1}$, Fazal Rabbi ${ }^{1}$, Muhammad Musharaf ${ }^{1}$, Muhammad Asad Bilal Awan ${ }^{1,2}$, Rizwan A. Memon ${ }^{1}$, Asif R. Rathore ${ }^{1}$, Pervaiz A. Chaudry ${ }^{1}$

1. Cardiac Surgery, National Institute of Cardiovascular Diseases, Karachi, PAK 2. Cardiac Surgery, College of Physicians and Surgeons Pakistan, Karachi, PAK

Corresponding author: Pervaiz A. Chaudry, pchaudhrymd1@gmail.com

\section{Abstract}

\section{Introduction}

The aim of this research is to evaluate the in-hospital and early outcomes of the first 100 adult cardiac surgeries performed at a newly developed satellite center in Sukkur, Pakistan.

\section{Methods}

This is an audit of the first 100 adult cardiac surgeries performed at a newly developed satellite center of the National Institute of Cardiovascular Diseases (NICVD) at Sukkur, Pakistan, from March 2018 to November 2018 with 12 months of post-operative follow-up. Patients were offered off-pump coronary artery bypass (OPCAB), on-pump coronary artery bypass (ONCAB), mitral valve replacement (MVR), aortic valve replacement (AVR), minimally invasive cardiac surgery (MICS), and congenital adult congenital heart disease (ACHD) procedures by expert faculty of NICVD with a minimum of five years of post-fellowship experience.

\section{Results}

The mean age was $47.11 \pm 14.6$ years, with a male predominance of $77 \%$. Hypertension and smoking were the most common risk factors that were observed in $32 \%$ and $33 \%$, respectively, followed by diabetes and dyslipidemia with a frequency of $20 \%$ and $9 \%$, respectively. The mean EuroSCORE (European System for Cardiac Operative Risk Evaluation) II for this patient cohort was $1.165 \pm 0.50$, with a maximum score of 2.3 in one patient. Out of 100 procedures, 51 were ONCAB, 19 were OPCAB, 16 were MVR, three were AVR, nine were ACHD, and two were MICS. Survival status post-operative as well as after one year was $100 \%$. The frequency of post-operative bleeding was $7 \%$, mean post-operative mechanical ventilation time was $213 \pm$ 273 hours, and in-hospital stay was $5.41 \pm 0.165$ days. Lost to follow-up at one year was $4 \%$ (four). During the follow-up assessment, $39.5 \%$ of the patients had complained of mild-to-moderate intensity retrosternal pain and $4.2 \%$ had superficial surgical site infection of the sternal wound. A significant improvement in functional class was observed in $38.5 \%$ of patients, whereas $4.2 \%$ (four) had a significant drop in functional

Received 03/18/2020 Review began 03/21/2020 Review ended 05/12/2020 Published 06/07/2020

\section{() Copyright 2020}

Zia et al. This is an open access article distributed under the terms of the Creative Commons Attribution License CC-BY 4.0., which permits unrestricted use, distribution, and reproduction in any medium, provided the original author and source are credited. class post-operatively.

\section{Conclusion}

Providing tertiary care and early cardiac surgical facility to the people of Sukkur at their doorstep, in a newly developed satellite center, has resulted in improved outcomes, early quality treatment facility, and avoidance of long travel time.

Categories: Cardiac/Thoracic/Vascular Surgery, Cardiology, Miscellaneous

Keywords: nicvd, cardiovascular diseases, cardiac surgery, pakistan, satellite center, sukkur, mics, opcab, off-pump coronary artery bypass (opcab), on-pump coronary artery bypass (oncab)

\section{Introduction}

The incidence of cardiovascular diseases has markedly increased in Pakistan for the last few years, and the hidden portion of the iceberg is growing day by day. Even the latest reported incidence of coronary artery disease and rheumatic heart disease requiring surgery in Pakistan dates back a decade [1-4].

Cardiac surgery is a field in which there is a high concentration of patients in the metropolitan area because the surgery is mainly carried out in medical institutions located in the metropolitan area. Due to this, policies must be developed in order to enhance the quality of healthcare and the geographical accessibility for patients residing in remote regions [5]. 
The need to introduce such centers at areas of the Sindh province came up due to high flow of cardiac patients traveling to Karachi for cardiac intervention or surgery, with more than 400 patients waiting for cardiac surgery at the National Institute of Cardiovascular Diseases (NICVD), as per the Karachi Clinics Registry, before the start of Sukkur, Larkana, and Tando Mohammad Khan (TMK) surgical centers. This figure has been markedly brought down now, so have earlier admissions, reduced traveling time, regular follow-up visits, and cost burden to the patient. The idea of establishing regional cardiac surgery centers has been reported in South Korea to meet the patient's requirements [5]. Also, the training requirement of more and more young cardiac surgeons can be effectively completed as the United States reports shortage of cardiothoracic surgeons by 2020 [6].

The inauguration of this 300-bed state-of-the-art satellite center in Sukkur took place on February 24, 2018. This center provides services such as modern and well-equipped 24/7 cardiac emergency, Cath Lab, coronary care unit, consulting clinics, advanced diagnosis, adult and pediatric cardiology, echocardiography, coronary artery angioplasty, angiographies, and cardiac surgical facility.

With the inauguration of cardiac surgery equipped satellite facilities by NICVD at Sukkur, Larkana, and TMK, there has been a lot of ease for people to get prompt cardiac care.

The first cardiac surgery at NICVD Sukkur took place in March 2018. Since then, this center has been providing complete cardiac surgical coverage to the population of Sukkur city and surrounding areas. To date, more than 500 cases have been operated at Sukkur, whereas this study only reports the starting of this newly developed center.

\section{Materials And Methods}

This retrospective observational study is an audit of the first 100 cases of cardiovascular surgeries performed at a newly developed satellite center of the NICVD at Sukkur from March 2018 to November 2018 with 12 months of post-operative follow-up.

Patients selected for any procedure met the American Heart Association guidelines criteria and had undergone heart team approval as per hospital policy before undergoing cardiac surgery. Pre-operative informed consent was taken from the patient and family. The European System for Cardiac Operative Risk Evaluation (EuroSCORE) II online calculator was used for risk stratification of patients [7]. All the patients electively admitted for surgery were offered guideline-directed general pre-operative measures to limit perioperative complications [8].

Patients were offered off-pump coronary artery bypass (OPCAB), on-pump coronary artery bypass (ONCAB), mitral valve replacement (MVR), aortic valve replacement (AVR), minimally invasive cardiac surgery (MICS), and congenital procedures by expert faculty of NICVD with a minimum of five years of post-fellowship experience.

OPCAB was performed using OctoBase ${ }^{\circledR}$ sternal retractor, Octopus ${ }^{\circledR}$ stabilizer, Urchin positioner ${ }^{\circledR}$, and AccuMist ${ }^{\circledR}$ Blower (all by Medtronic, Minneapolis, MN, USA). OPCAB myocardial protection was performed using intracoronary shunts size ranging from 1.0 to $2.0 \mathrm{~mm}$ with the left internal mammary artery (LIMA) to the left anterior descending artery (LAD) as the first strategy. ONCAB and valvular procedures were performed through median sternotomy with target activated clotting time above 480 seconds and myocardial protection using St. Thomas cardioplegia delivered antegrade (graft plegia was preferred by few surgeons) or retrograde with topical cooling. Minimally invasive atrial septal defect (ASD) closure was performed using the same technique as described in our previous publication on double valve replacement with central cannulation [9].

After discharge, all the patients were scheduled for routine follow-up visits at the cardiac surgical clinic of NICVD Sukkur at least four times, i.e., after one week, one month, six months, and one year after the procedure. They were also contacted by cell phone as a reminder for their follow-up visit. Early postoperative echocardiography was performed on the first post-operative day and before discharge, whereas follow-up echocardiography was performed in all patients after six months of the procedure. Patients requiring vitamin $\mathrm{K}$ antagonists (VKA) were managed in the INR (international normalized ratio) clinic. All patients were referred to the rehabilitation center for early recovery. Patients requiring emergent medical attention were asked to report to the NICVD emergency room or clinic at any time.

During their hospital stay, the patients were kept under observation, and in-hospital (30 days postoperative) outcomes were assessed, which included mortality, morbidity, length of stay, ventilation time, post-operative bleeding, reoperation, and re-intubation.

At one-year follow-up, survival status, complaints, infection (superficial or deep), function class, echocardiographic assessment, and recurrence of disease were assessed. Data analysis was carried out using SPSS Statistics for Windows, Version 21.0 (IBM Corp., Armonk, NY, USA). 


\section{Cureus}

\section{Results}

The mean age of the first 100 patients' undergone cardiovascular surgeries at this satellite center was $47.11 \pm$ 14.6 years, with a male predominance of $77 \%$. Hypertension and smoking were the most common risk factors which were observed in $32 \%$ and $33 \%$, respectively, followed by diabetes and dyslipidemia with a frequency of $20 \%$ and $9 \%$, respectively. The mean EuroSCORE II for this patient cohort was $1.165 \pm 0.50$, with a maximum score of 2.3 in one patient. Mean ejection fraction (EF) was $56.33 \pm 6.96 \%$. Distribution of baseline demographic and clinical characteristics of the patients by gender are presented in Table 1 .

\begin{tabular}{|c|c|c|}
\hline Characteristics & Males & Females \\
\hline Total & 77 & 23 \\
\hline Age, years & $50 \pm 13$ & $36 \pm 13$ \\
\hline Weight, kg & $73 \pm 14$ & $59 \pm 19$ \\
\hline \multicolumn{3}{|l|}{ NYHA classification } \\
\hline I & $3.9 \%(3)$ & $6.5 \%(5)$ \\
\hline II & $42.9 \%(33)$ & $6.5 \%(5)$ \\
\hline III & $44.2 \%(34)$ & $15.6 \%(12)$ \\
\hline IV & $9.1 \%(7)$ & $1.3 \%(1)$ \\
\hline \multicolumn{3}{|l|}{ Co-morbidities } \\
\hline Diabetics & $24.7 \%(19)$ & $1.3 \%(1)$ \\
\hline Hypertension & $40.3 \%(31)$ & $1.3 \%(1)$ \\
\hline Dyslipidemia & $10.4 \%(8)$ & $1.3 \%(1)$ \\
\hline Smoking & $41.6 \%(32)$ & $1.3 \%(1)$ \\
\hline Cardiogenic shock & $6.5 \%(5)$ & $2.6 \%(2)$ \\
\hline Ejection fraction & $56.32 \pm 7.14$ & $56.35 \pm 6.50$ \\
\hline EuroSCORE II & $1.23 \pm 0.49$ & $0.97 \pm 0.5$ \\
\hline
\end{tabular}

TABLE 1: Baseline demographic and clinical characteristics of the patients by gender

NYHA, New York Heart Association; EuroSCORE, European System for Cardiac Operative Risk Evaluation

A majority of the smokers, 36.3\% (12/33), had a history of more than one pack a year. These patients underwent breathing exercises, chest physiotherapy, and spirometry before surgery and pulmonary function test assessment for the prediction of future outcomes. Among diabetic patients, 65\% (13/20) had uncontrolled insulin-dependent diabetes mellitus, whereas $15 \%(3 / 20)$ had an incidental diagnosis of diabetes after pre-operative routine laboratory investigations. Mean HbA1c of diabetic patients was $9.85 \pm$ $2.0 \%$; therefore, surgery was considered once this figure was brought around 7.5. Perioperative dose calculated insulin infusion was used in these patients during the course of surgery with guideline-directed target blood sugar. Frequency distribution of the types of procedures is presented in Figure 1 . 


\section{Cureus}

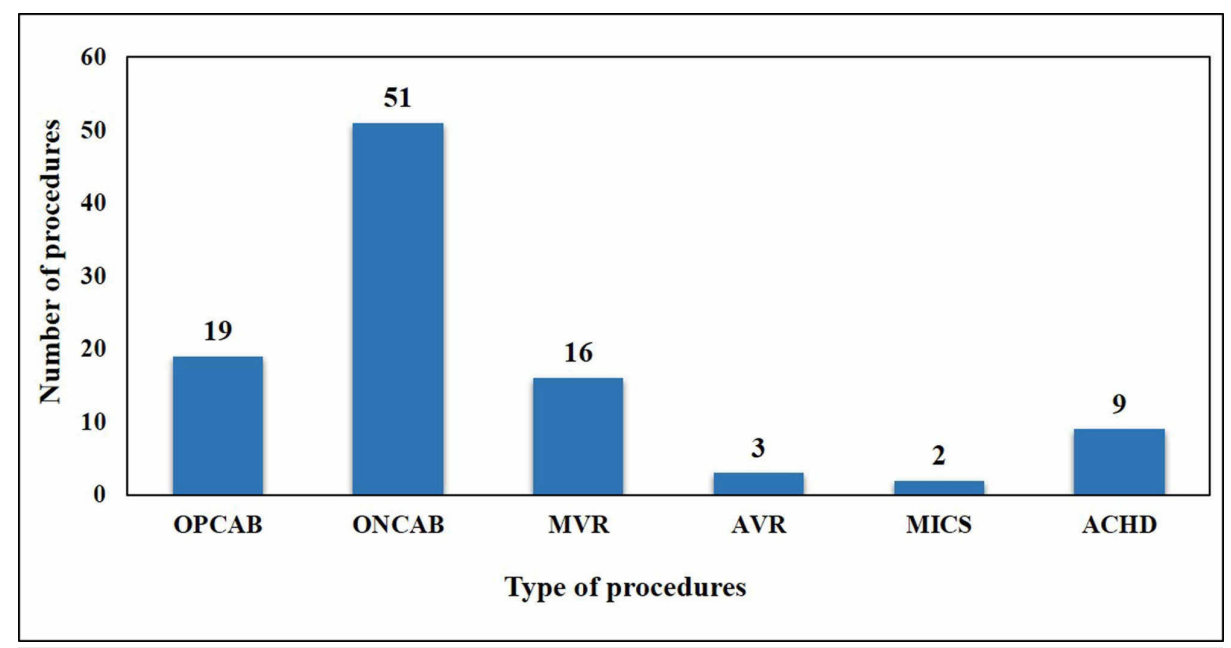

\section{FIGURE 1: Frequency distribution of types of first 100 procedures performed at Sukkur satellite center}

OPCAB, off-pump coronary artery bypass; ONCAB, on-pump coronary artery bypass; MVR, mitral valve replacement; AVR, aortic valve replacement; MICS, minimally invasive cardiac surgery; ACHD, adult congenital heart disease procedures

A total of 70 coronary artery bypass grafting (CABG) surgeries were performed out of which 7 were operated as emergency cases due to signs of cardiogenic shock, whereas the rest of the patients were electively operated and admitted through the outpatient department. Nineteen (27.1\%) of CABG surgeries were OPCAB, whereas the remaining $72.9 \%$ (51/70) were conventional ONCAB surgeries. Our primary goal remained completely revascularization, with the LIMA as a preferred conduit for the LAD to improve survival benefit for the patient. We achieved a mean number of distal grafts as $3.01 \pm 0.577$, including 11 patients in the CABG group who had two distal targets for anastomosis. Detailed frequency of types of procedures against the subsequent diagnosis and post-operative outcomes are presented in Table 2. 


\section{Cureus}

Diagnosis and procedures

\section{CABG}

Triple-vessel coronary artery disease

Two-vessel coronary artery disease

Left main with triple-vessel coronary artery disease

Aortic valve replacement (mechanical valves)

Severe symptomatic aortic regurgitation

Mitral valve replacement (mechanical valves)

Severe symptomatic mitral stenosis

Severe symptomatic mixed mitral disease

Minimally invasive and conventional atrial septal defect closure

Secundum atrial septal defect

Conventional ventricular septal defect closure

Ventricular septal defect

Pulmonary valvotomy

Isolated pulmonary stenosis

Post-operative outcomes

Mortality

CVA

Low cardiac output state

Renal dysfunction

Post-operative bleeding

Re-intubation

TABLE 2: : Diagnosis, procedures, and post-operative outcomes of the first 100 procedures performed at Sukkur satellite center

CABG, coronary artery bypass grafting; CVA, cerebrovascular accident

Out of 70 patients, 65 (93\%) had LIMA anastomosed to LAD, in 4 patients LIMA was not used considering comorbidities and life expectancy, and 1 patient had an operative injury to LIMA. MVR surgeries were performed in 16 patients, AVR in 3 , adult congenital heart surgery in 11, out of which 2 patients were offered minimally invasive right anterior mini-thoracotomy ASD closure.

The frequency of surgically significant post-operative bleeding was observed in seven (7\%) of the patients, out of which three patients were surgically managed, whereas the rest of the patients had disturbed coagulation profile and were therefore managed on medical therapy. Mean post-operative mechanical ventilation time remained $213 \pm 273$ hours, which also included total mechanical ventilation time for complicated patients requiring prolonged ventilation and those who had re-intubation. In-hospital stay 


\section{Cureus}

remained $5.41 \pm 0.165$ days, with $0 \%$ in-hospital mortality.

Three patients underwent immediate reoperation. One was diagnosed with perioperative cardiac tamponade, and, therefore, emergent resternotomy in the intensive care unit (ICU) was performed to relieve the tamponade; afterward the patient was moved to the operating room. Two patients had perioperative massive bleeding, which were managed in the operating room

Intra-aortic balloon pump (IABP) was electively placed in six patients undergoing CABG, whereas one patient had an emergent IABP placement because of post-operative low cardiac output state (LCOS). The patient who developed LCOS underwent re-intubation as well because of secondary respiratory failure as well. Along with that, another patient was re-intubated who had post-operative renal dysfunction requiring dialysis. One patient had post-operative ischemic stroke. All of these complicated patients requiring a multidisciplinary approach were managed by intensivists, nephrologists, and neurologists.

Lost to follow-up at one year was $4 \%$ (four), and follow-up of the remaining 96 (96\%) patients was completed successfully. During the follow-up assessment, 39.5\% (38/96) patients had complained of mildto-moderate intensity retrosternal pain with numbness over the left parasternal area, $4.2 \%$ (four) had superficial surgical site infection of the sternal wound, and one patient had a sternal dehiscence and underwent rewiring for sternal closure. A significant improvement in functional class was observed in $38.5 \%$ (37/96) of the patients, whereas $4.2 \%$ (four) had a significant drop in functional class, for which all of these patients had complications during the perioperative period. One of the patients had dropped in EF from $45 \%$ to $35 \%$ due to early graft occlusion and underwent percutaneous coronary intervention for disease recurrence. Survival status at one-year follow-up remained $100 \%$. On-year follow-up status of patients is presented in Table 3. 


\section{Cureus}

\section{One-year follow-up}

N

Survival status $100 \%(96)$

Complaints

Pain $39.6 \%(38)$

Cough $36.5 \%(35)$

Serous discharge $24 \%(23)$

Muscle cramps $18.8 \%(18)$

Sleeplessness

Anxiety

Immobility

Infection

Superficial (SSI) sternal

Superficial (SSI) leg

Deep sternal wound infection

Sternal dehiscence

Function class

Deterioration

Improvement

Echocardiographic assessment

Deterioration ejection fraction

Severe pericardial effusion

Structural valvular deterioration

$4.2 \%(4)$

Paravalvular leak

$0 \%(0)$

Recurrence of disease

$1 \%(1)$

TABLE 3: One-year follow-up status of the first 100 procedures performed at Sukkur satellite center

SSI, surgical site infection

\section{Discussion}

Closed heart operations in Pakistan began by the native surgeons in the late 1950s at a few centers in Karachi, Lahore, and Rawalpindi. But open-heart operations began by Dr. Donald Edward Bowes, a Canadian born missionary surgeon in 1967-1968 at United Christian Hospital Lahore [10]. Regular civilian cardiac surgical program began in Pakistan with the establishment of the NICVD at Karachi in the early 1970s [11]. Since then, NICVD has remained the center of excellence to provide quality care to cardiac patients.

In the Third World, cardiac patients often suffer from not only the nature of their illnesses but also the insufficiency of the facilities available [12]. There has been a marked rise in the patients requiring cardiac surgery, and Pakistan has a very limited number of government-funded centers to timely operate on these patients. Therefore, the only way out remained to enlist these patients and make them wait for their surgery. This resulted in a lot of morbidity, out-of-hospital cardiac arrest (OHCA) events, and mortality in these patients waiting for their turn to undergo coronary bypass or valve-related surgery because of lack of rural cardiac facilities. Therefore, expanding the cardiac surgical coverage remained a primary focus for health care providers. Till date, more than 500 successful cardiac surgeries have been performed at NICVD 
Sukkur. With an aim to minimize the time required by a patient to avail early cardiac care, the team of NICVD is moving forward to provide a near-to-door cardiac facility across Pakistan.

We have faced a huge burden of diabetics, hypertension, and smoking in the Sukkur population, predicting a higher risk for adverse outcomes. But we have remained successful to prove that timely surgical intervention, adequate pre-operative management, and regular follow-up are keys to successful outcomes at a newly developed cardiac surgery institute. Institutional results are essential to enable surgeons to determine whether international data are in keeping with local findings [13]. NICVD Sukkur being first of its kind government-funded state of the art cardiac surgical facility has demonstrated impressive surgical results compared with reliable Western standards.

NICVD being a non-private fully funded government organization regards maximum post-operative management protocols with zero financial burdens on families. This facility has provided all advanced resources such as standardized medications, IABP, continuous renal replacement therapy machine, automatic implantable cardiac defibrillators, ventricular assist devices, and extracorporeal membrane oxygenation. This means that patients who suffer from a poor post-operative recovery did receive the most optimal possible support. This should encourage other hospitals in the developing world to aspire to high levels of care.

\section{Conclusions}

With an increasing incidence of patients requiring cardiac surgeries, the inauguration of new cardiac surgical centers is a necessity in Pakistan. Establishment of the Sukkur satellite center has allowed for the provision of quality cardiac surgical care. Despite being a newer unit, it experienced faculty backup has resulted in enormous pre- and post-operative management with minimum reported complications and zero mortality in the first 100 cases during the in-hospital period.

\section{Additional Information \\ Disclosures}

Human subjects: Consent was obtained by all participants in this study. National Institute of Cardiovascular Diseases, Karachi issued approval ERC-11/2020. This study was approved by the Ethical Review Committee of the National Institute of Cardiovascular Diseases (NICVD), Karachi, Pakistan. Animal subjects: All authors have confirmed that this study did not involve animal subjects or tissue. Conflicts of interest: In compliance with the ICMJE uniform disclosure form, all authors declare the following: Payment/services info: All authors have declared that no financial support was received from any organization for the submitted work. Financial relationships: All authors have declared that they have no financial relationships at present or within the previous three years with any organizations that might have an interest in the submitted work. Other relationships: All authors have declared that there are no other relationships or activities that could appear to have influenced the submitted work.

\section{References}

1. Rizvi SF, Khan MA, Kundi A, Marsh DR, Samad A, Pasha O: Status of rheumatic heart disease in rural Pakistan. Heart. 2004, 90:394-9. 10.1136/hrt.2003.025981

2. Omran AR: The epidemiologic transition. A theory of the epidemiology of population change . Milbank Mem Fund Q. 1971, 49:509-38.

3. Murray CJL, Lopez AD: Mortality by cause for eight regions of the world: Global Burden of Disease Study . Lancet. 1997, 349:1269-76. 10.1016/S0140-6736(96)07493-4

4. Treasure T: Advances in cardiac surgery. Practitioner. 2001, 245:422-4.

5. Park CS, Park NH, Sim SB, et al.: Development of models for regional cardiac surgery centers . Korean J Thorac Cardiovasc Surg. 2016, 49:28-36. 10.5090/kjtcs.2016.49.S1.S28

6. Grover A, Gorman K, Dall TM, et al.: Shortage of cardiothoracic surgeons is likely by 2020 . Circulation. 2009, 120:488-94. 10.1161/CIRCULATIONAHA.108.776278

7. Nashef SA, Roques F, Sharples LD, Nilsson J, Smith C, Goldstone AR, Lockowandt U: EuroSCORE II. Eur J Cardiothorac Surg. 2012, 41:734-45. 10.1093/ejcts/ezs043

8. Sousa-Uva M, Head SJ, Milojevic M, et al.: 2017 EACTS Guidelines on perioperative medication in adult cardiac surgery. Eur J Cardiothorac Surg. 2018, 53:5. 10.1093/ejcts/ezx314

9. Zia K, Mangi AR, Bughio H, Tariq K, Chaudry PA, Karim M: Initial experience of minimally invasive concomitant aortic and mitral valve replacement/repair at a tertiary care cardiac centre of a developing country. Cureus. 2019, 11:e5707. 10.7759/cureus.5707

10. Hosain N: The early days of cardiac surgery in South Asia: the history and heritage . Ann Thorac Surg. 2017, 104:361-6. 10.1016/j.athoracsur.2017.01.039

11. Hosain N, Amin F, Rehman S, Koirala B: Know thy neighbors: the status of cardiac surgery in the South Asian countries around India. Indian Heart J. 2017, 69:790-6. 10.1016/j.ihj.2017.09.219

12. Barolia R, Ali F, Jaffar S: Coronary artery bypass grafting: quality of life of patients in Karachi . Br J Nurs. 2012, 21:349-55. 10.12968/bjon.2012.21.6.349

13. Sheikh MR, Khan MS, Saeed Z, Furnaz S, Sharif H: Outcome of coronary artery bypass grafting in a tertiarycare center in Pakistan. Asian Cardiovasc Thorac Ann. 2015, 23:276-81. 10.1177/0218492314545620 